\title{
An Entropy Method For Multisource Data Fusion
}

\author{
B. Fassinut-Mombot \\ J.B. Choquel \\ Laboratoire d'Analyse des Système du Littoral (UPRES-EA2600) \\ Université du Littoral Côte d'Opale (ULCO) \\ 195, Rue du Pasteur Martin-Luther-King, BP 649 \\ 62228 Calais Cedex, FRANCE \\ Bienvenu.Fassinut-Mombot@lasl-gw.univ-littoral.fr \\ Jean-Bernard.Choquel@lasl-gw.univ-littoral.fr
}

\begin{abstract}
The present paper proposes a generic model of the multisource data fusion in the framework of the theory of information, with closer attention being given the different nature of data processed in common cases. This model that we have called entropy model is then used to elaborate processing methods able to face specific problems that may arise when multisource systems are implemented to achieve functions like classification and pattern recognition, matching of ambiguous observations, estimation, detection or tracking. Crucial practical problems to data fusion are more specifically dealt with, such as information representation, appropriate combination processing and decision making. Some clues are given on the practical use and implementation of such an approach, for example, in the distributed estimation problem.
\end{abstract}

Keywords: Multisource data fusion, Bayes rule, Maximum entropy, Shannon mutual information, Entropyadaptive-aggregation algorithm.

\section{Introduction}

The objective of multisource data fusion is to minimize the impact of uncertainties and get the most information out of the sources. The potential advantages of integrating multiple source information correspond to the notion of redundancy, complementarity, flow and cost of the information, respectively [1]. Generally speaking, a multisource data fusion approach involves three components: information representation (modelization), uncertainty description (combination) and optimization method (decision making). Information representation is referred to as choosing the best format and the best level of detail to represent information of interest, while uncertainty description addresses the way we describe uncertainty of information. Optimization is needed either to maximize overall information or to minimize overall uncertainty according to some criteria.

In a previous paper, Fassinut-Mombot and all. [2] have shown how we can use entropy criterion to solve optimal information fusion problem. They have also shown that the formulation of Shannon's conditional entropy is required the computation of the prior probabilities and conditional probabilities. The present paper is essentially established to offer a natural setting for modeling information stemming from various sources (for instance, several experts, sensors, databases), for missing these pieces of information, and then for taking a decision. Our objective is to develop an efficient algorithm for solving the multisource data fusion problem. This algorithm, which is based on ideas from Information Theory and Bayes rule for statistical inference, is inherently probabilistic. The entropy of a system as defined by Shannon $[3,4]$ gives a measurement of ignorance about its actual structure, and offers a framework for measuring the performance or for characterizing a system. Based on Shannon's entropy concept, researchers have proposed many different methods for modeling uncertainty and imprecision.

In fact, De Luca and Termini in [5] were pioneered (for us) in modelization of uncertainty linked to the description of an object in terms of informational measurements. Couso and others [6] have shown the relationship between fuzzy measurement and information measurement. Fioretto and Sgarro [7] used the advantages of maximum entropy for defining confidence measurement of believes theory. And more recently, Zhou and Leung [8] have presented the minimun entropy fusion approach for multisource data fusion in non-Gaussian environments. Others researchers have proposed the utilization of information measurement applied in learning problems [9], in Structural Analysis of complex systems [10], [11] or in detection systems [12].

The rest of this paper is organized as follows: Section 2, after having specified contours of the problem of fusion that we will study, we propose the theoretic formalism, as described in [10], [11], to solve the multisource data fusion problem. We then show the importance of the modelization of knowledge for the optimality of the fusion rule [13]. In section 3 , we consider the probability distributions estimation problem 
by using the principle of maximum entropy, which provides stronger justification for resulting optimization method. Also, we give, in section 4, the maximum entropy algorithm for modeling the observations and we propose an algorithm that we called entropy adaptive aggregation algorithm (EA2 algorithm) for multisource data fusion problem with a demonstrated potential to obtain optimum of the entropy criterion (sufficient condition of optimality). An illustration of entropy model, through the application of distributed estimation system with data fusion, is provided in section 5. Finally, Section 6 is our brief conclusion and future work.

\section{Entropy model for multi- source data fusion}

The Information Theory (IT) was first introduced by Shannon and others [3], [4] in 1948, known initially for the transmission study of the messages transmitted by a source with a receiver through a channel (Hertzian) subject to the noise. The use of IT within the framework of the Structural Analysis of the complex systems permitted to model the knowledge that we have on the state of a system. It also permits to rigorously model our lack of knowledge of this statement, through many clues. The familiar readers about the Structural Analysis will note that this paper is strongly inspired of the references [10], [11], to formalize the resolution of the fusion problem.

\subsection{Preliminaries}

The problem of fusing multisource data we consider is the following. Consider a multisource fusion system with $N(N \geqslant 2)$ sources as shown in Figure 1 . Let $\Omega$ a population compound of individuals divided into $K$ classes, (or semantic objects), plus one unknown class (ignorance hypothesis $\theta) Y=\left\{c_{1}, c_{2}, \ldots, c_{K}, \theta\right\}$, mutually exclusive and exhaustive (closed environment), that explain the state (the observation) of the system:

$$
\forall l \neq k, \quad P\left(c_{l}, c_{k}\right)=0 \quad \text { and } \quad \sum_{k=1}^{K+1} P\left(c_{k}\right)=1
$$

where $P\left(c_{l}, c_{k}\right)$ and $P\left(c_{k}\right)$ are respectively joint probabilities and prior probabilities of system. Multiple sources observe parametric data from an entity whose identity is unknown. Each of the sources provides an identity declaration, or hypothesis about the object's identity that note $X^{i} \triangleq\left\{X_{j}^{i}\right\}_{j=1}^{M}$, compound of $M$ modalities quantitative, qualitative or structured. Thus, a source can be represented by a sensor, an expert or a database.

Let $\omega$ the object (or the entity) present in $\Omega$ on which relates the decision. The set of the observations available on $\Omega$ is gathered in the vector of observations $X \triangleq\left\{\left\{X_{j}^{i}\right\}_{j=1}^{M}, i=1, \ldots, N\right\}$ on which is based fusion, via measurements $M_{k}^{i}(\omega)$, connecting information on $\omega$ provided by source $X^{i}$ about a potential decision

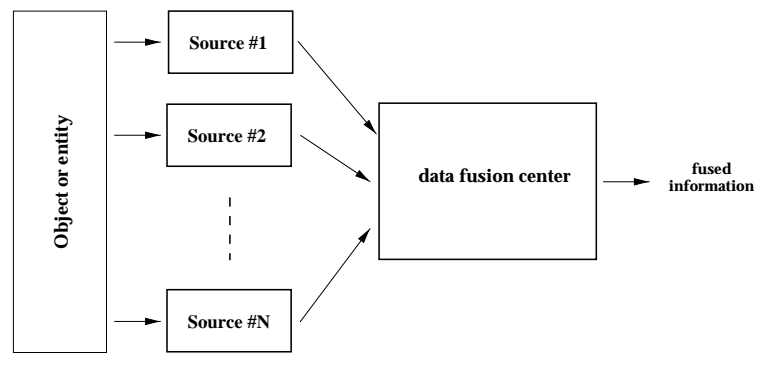

Figure 1: Multisource fusion system

$c_{k}$ on $\omega$ (see Table 1$)$. This information may be imprecise, erroneous, incomplete, or poor to the problem. The decision about $\omega$ consists in affecting an hypothesis $c_{k}$ of $Y$ to it.

Table 1: Measurements table

\begin{tabular}{|c|c|c|c|c|c|c|}
\hline \multicolumn{7}{|c|}{ Object } \\
\hline & $c_{1}$ & $c_{2}$ & $c_{3}$ & $\cdots$ & $c_{K}$ & $\theta$ \\
\hline$X^{1}$ & $M_{1}^{1}$ & $M_{2}^{1}$ & $M_{3}^{1}$ & $\cdots$ & $M_{K}^{1}$ & $M_{K+1}^{1}$ \\
\hline$X^{2}$ & $M_{1}^{2}$ & $M_{2}^{2}$ & $M_{3}^{2}$ & $\cdots$ & $M_{K}^{2}$ & $M_{K+1}^{2}$ \\
\hline$\vdots$ & $\vdots$ & $\vdots$ & $\vdots$ & $\cdots$ & $\vdots$ & $\vdots$ \\
\hline$X^{N}$ & $M_{1}^{N}$ & $M_{2}^{N}$ & $M_{3}^{N}$ & $\cdots$ & $M_{K}^{N}$ & $M_{K+1}^{N}$ \\
\hline
\end{tabular}

It is then acts to combine by an operator $F$ overall relative measurements the same decision $c_{k}$ issued from various sources (columns of the table 1 ), to obtain the fused measurement $X_{k}^{*}=F\left(M_{k}^{i}, 1 \leqslant i \leqslant N\right)$. Then, to find a function of decision $D: X_{k}^{*} \longmapsto$ $\left\{c_{1}, c_{2}, \ldots, c_{K}, \theta\right\}$ such as $D \circ X_{k}^{*}$ is a good approximation of $Y$.

\subsection{Information representation}

Information representation is referred to as choosing the best format and the best level of detail to represent information of interest. The theory most exploited in the literature is by far the probability theory, associated with the bayesian theory of the decision [14]. Information is here characterized by a conditional probability distibution, in our case, the probability that a particular class $c_{k}$ is the class of the object $\omega$, being given information issued from various sources. By using the Bayes rule for the conditional probabilities:

$$
P\left(\omega \in c_{k} / X^{i}\right) \triangleq \frac{P\left(X^{i}, c_{k}\right)}{\sum_{k=1}^{K+1} P\left(X^{i}, c_{k}\right)}
$$

information can be thus modeled by joint probabilities $P\left(X^{i}, c_{k}\right)$ of simultaneous realization of an observation $X^{i}$ and of a particular class $c_{k}$. Then, the measurement $M_{k}^{i}(\omega)$ takes the following form (see Table 


$$
\begin{aligned}
M_{k}^{i}(\omega) & =P\left(X^{i}(\omega), c_{k}\right) \\
& =\prod_{j=1}^{M} P\left(X_{j}^{i}(\omega), c_{k}\right)
\end{aligned}
$$

The assumption being that all observations $X_{j}^{i}(\omega)$ are conditionally independent, that is independent with $\omega$ belongs to $c_{k}$ (assumption known as i.i.d.). In order to obtain the joint probabilities $P\left(X^{i}(\omega), c_{k}\right)$, we start from the principle that these probabilities can be estimated by a parametric or non-parametric method. In fact, an interpretation frequentist of the probabilities, as it is the case in Structural Analysis, where it counts the number of occurrences of an event is not always acceptable for the purpose of modeling a single event. Moreover, the estimate of the probabilities using the relative frequencies (or occurrence) require that, at the time of the training, of the very strict constraints are verified by the system (ergodicity, stationnarity) and the population of training $\left(\operatorname{card}(\Omega) \geqslant \prod_{i=1}^{N}\left(\operatorname{card}\left(X^{i}\right)-1\right)\right)[15]$.

Table 2: Contingency table

\begin{tabular}{|c|c|l|c|c|}
\hline \multicolumn{5}{|c|}{ Object } \\
\hline & $c_{1}$ & $\cdots$ & $c_{K}$ & $\theta$ \\
\hline$X^{1}$ & $P\left(X^{1}, c_{1}\right)$ & $\cdots$ & $P\left(X^{1}, c_{K}\right)$ & $P\left(X^{1}, c_{K+1}\right)$ \\
\hline$X^{2}$ & $P\left(X^{2}, c_{1}\right)$ & $\cdots$ & $P\left(X^{2}, c_{K}\right)$ & $P\left(X^{2}, c_{K+1}\right)$ \\
\hline$\vdots$ & $\vdots$ & $\cdots$ & $\vdots$ & $\vdots$ \\
\hline$X^{N}$ & $P\left(X^{N}, c_{1}\right)$ & $\cdots$ & $P\left(X^{N}, c_{K}\right)$ & $P\left(X^{N}, c_{K+1}\right)$ \\
\hline
\end{tabular}

\subsection{Data combination}

Once information issued from various sources is modeled, it can be combined according to a rule based on the minimization of the conditional entropy [2]. In fact, the Shannon conditional entropy $H(Y / X)$, where $Y$ is the vectorial variable corresponding to the class of membership and $X$ the vectorial variable corresponding to the vector of observations, is a measurement of uncertainty about $Y$, when the information provided by the observations vector $X$ is known.

The combination rule of entropy consists in solving a problem of optimization, that is to find the optimal partition $X^{*}$ such as:

$$
X^{*}=\arg \min _{X^{\prime} \in \mathbb{P}(X)}\left\{H\left(Y / X^{\prime}\right)\right\}
$$

where $\mathbb{P}(X)=\left\{X^{\prime}: X^{\prime} \subseteq X\right\}$

$$
\begin{aligned}
H\left(Y / X^{\prime}\right) \triangleq & H\left(X^{\prime}, Y\right)-H\left(X^{\prime}\right) \\
= & -\sum_{i=1}^{N} P\left(X^{\prime}=X^{i}\right) \cdot H\left(Y / X^{\prime}=X^{i}\right) \\
= & -\sum_{i=1}^{N} P\left(X^{\prime}=X^{i}\right) \\
& \times\left[\sum_{k=1}^{K+1} P\left(c_{k} / X^{i}\right) \log P\left(c_{k} / X^{i}\right)\right]
\end{aligned}
$$

$\operatorname{logs}$ are chosen to the base 2, say; $0 \log 0$ is set equal to 0 . Expression 6 can be write again:

$$
H\left(Y / X^{\prime}\right)=E_{X^{\prime}}\left[H\left(Y / X^{\prime}=X^{i}\right)\right]
$$

which represents the expectation of partial conditional entropy.

The aim set by the choice of such criterion is to be able to minimize the complexity of combination of information (or measurements). In fact, certain information provided can be redundant and/or complementary for the fusion problem, or can embed the system under a stream of too expensive information to manage. The minimization of the conditional entropy $H\left(Y / X^{\prime}\right)$ thus permits to lead to a decision about the object $\omega$ as pertinent as using the set of measurements $M_{k}^{i}(\omega)$ of the contingency table (see Table 2). In conclusion, taking into account the model of fusion chosen, the combination entropy rule is written:

$$
\forall c_{k} \in Y, \quad X_{k}^{*}=\arg \min _{X^{\prime} \in \mathbb{P}(X)}\left\{H\left(c_{k} / X^{\prime}\right)\right\}
$$

where

$$
\begin{aligned}
H\left(c_{k} / X^{\prime}\right)= & -\sum_{i=1}^{N} P\left(X^{\prime}=X^{i}\right) \cdot H\left(c_{k} / X^{\prime}=X^{i}\right) \\
= & -\sum_{i=1}^{N} P\left(X^{\prime}=X^{i}\right) \\
& \times\left[P\left(c_{k} / X^{i}\right) \log P\left(c_{k} / X^{i}\right)\right] \\
= & -\sum_{i=1}^{N} P\left(X^{i}, c_{k}\right) \log P\left(c_{k} / X^{i}\right)
\end{aligned}
$$

This fused measurement thus permit to characterize the pertinence of information (uncertainty and imprecision) provided by the various sources, relative to a particular class $c_{k}$.

\subsection{Decision making}

This last step of the fusion process relates the construction (by estimate generally) of the decision function $D$, which associates the class to which belongs an object $\omega$. This decision binary is accompanied by the definition of a clue of good decision which 
measures the quality of the decision, possibly being able to dismiss it [13].

On the basis of the principle which the minimization of the conditional entropy is equivalent to a minimization of the errors of classification, the rule of decision is written:

$$
\begin{aligned}
& \omega \in c_{k}, \text { if } \\
& H\left(c_{k} / X_{k}^{*}\right)=\min \left\{H\left(c_{l} / X_{l}^{*}\right), 1 \leqslant l \leqslant K+1\right\}
\end{aligned}
$$

This rule thus permits to characterize the pertinence of the decision $\omega \in c_{k}$, knowing that information available is reliable. To raise all ambiguities on this decision, we define a measurement of the quality of the decision by an informational clue called modelisability [10], and that we rewrite as follows:

$$
q\left(c_{k} / X_{k}^{*}\right)=\frac{H\left(c_{k}\right)-H\left(c_{k} / X_{k}^{*}\right)}{\sum_{l=1}^{K+1} H\left(c_{l}\right)-H\left(c_{l} / X_{l}^{*}\right)}
$$

where term $\sum_{l=1}^{K+1} H\left(c_{l}\right)-H\left(c_{l} / X_{l}^{*}\right)$ is interpreted as a measurement of conflict between hypothesis and model. This clue indicates the possibility of improving quality of the decision by blaming all the fusion process. It then poses the problem of the feasibility of the modeling of $c_{k}$ by $X_{k}^{*}$. In this direction, we propose a clue of good combination based, also, on an informational clue:

$$
m\left(c_{k} / X_{k}^{*}\right)=1-\frac{H\left(c_{k} / X_{k}^{*}\right)}{H\left(c_{k}\right)}
$$

We see that $m\left(c_{k} / X_{k}^{*}\right) \in[0,1]$ and represents the rate explicable of entropy of $c_{k}$. Where value 0 indicates that fused measurement $X_{k}^{*}$ does not bring any information about the class $c_{k}\left(\omega \in c_{k}\right.$, it is then an obviousness!). When $m(\cdot / \cdot)$ is equal to 1 the combination is perfected (ideal case). The large $m(\cdot / \cdot)$ (with $0 \leqslant m(\cdot / \cdot) \leqslant 1$ ) corresponds to the situations where information available is very incomplete and or is sullied with uncertainty. We can see that $m\left(c_{k} / X_{k}^{*}\right)$ is maximum when $H\left(c_{k} / X_{k}^{*}\right)$ is minimum.

The association of these two clues $m\left(c_{k} / X_{k}^{*}\right)$ and $q\left(c_{k} / X_{k}^{*}\right)$ is enough to entirely characterize the quality of the combination and decision rules. In conclusion, the decision rule is the following:

$$
\begin{aligned}
& \omega \in c_{k}, \text { if } \\
& \left\{\begin{array}{l}
H\left(c_{k} / X_{k}^{*}\right)=\min \left\{H\left(c_{l} / X_{l}^{*}\right), 1 \leqslant l \leqslant K+1\right\} \\
q\left(c_{k} / X_{k}^{*}\right)=\frac{H\left(c_{k}\right)-H\left(c_{k} / X_{k}^{*}\right)}{\sum_{l=1}^{K+1} H\left(c_{l}\right)-H\left(c_{l} / X_{l}^{*}\right)} \geqslant s
\end{array}\right.
\end{aligned}
$$

where $s$ is a threshold which remains to be determined in function of the application. Figure 2 illustrates the process of using a entropy model for multisource data fusion.

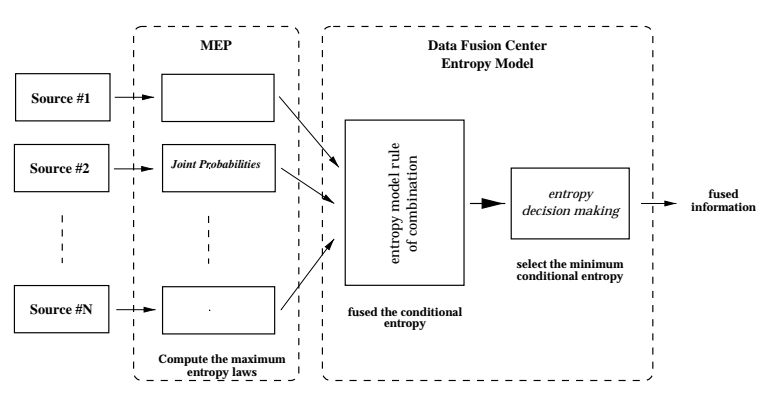

Figure 2: Summary of Entropy Model fusion

\section{Probabilities estimation}

As introduced above, we have chosen to model information issued from various sources, relatively with an object to be identified, by a parametric, rather than by learning on a training set of examples (relative frequencies).

What is it all about? For the purpose of assigning a probability law to a couple of variables $\left\{\left(X^{i}, c_{k}\right), i=1, \ldots, N ; k=1, \ldots, K+1\right\}$ on which we have a set of data $\left\{X_{j}^{i}\right\}_{j=1}^{M}$, observations on an object $\omega$, and a set of realization $c_{k} \in Y$ of a variable discrete which indicates the membership of the object to a particular class. A common practice for modeling uncertainty on a variable is to apply a maximum entropy principle (MEP), where we take as distribution of this variable the Shannon maximum entropy distribution, subject to some given constraints.

\subsection{Principle of maximum entropy}

The maximum entropy principle, denoted MEP or MaxEnt, is a procedure for inducing an unknown probability distribution starting from partial information (poor knowledge), that is verifying various empirical constraints defined using functions (or observables), denoted $\left\{\phi_{l}(\cdot), l=0, \ldots, L\right\}$.

In our case, to determine $P\left(X^{i}, c_{k}\right)$, the MEP is formulated as follows:

$$
\begin{aligned}
\widehat{P}\left(X^{i}, c_{k}\right)= & \arg \max _{P \in \mathcal{P}}\{H[P]= \\
& \left.-\sum_{k=1}^{K+1} \int P\left(X^{i}, c_{k}\right) \cdot \log P\left(X^{i}, c_{k}\right) d X^{i}\right\}
\end{aligned}
$$

where

$$
\begin{array}{r}
\mathcal{P}=\left\{P\left(X^{i}, c_{k}\right): \sum_{k=1}^{K+1} \int \phi_{l}\left(X^{i}, c_{k}\right) \cdot P\left(X^{i}, c_{k}\right) d X^{i}=\right. \\
\left.\frac{1}{M} \sum_{k=1}^{K+1} \sum_{j=1}^{M} \phi_{l}\left(X_{j}^{i}, c_{k}\right), l=0, \ldots, L\right\}
\end{array}
$$

with $\phi_{0}=1$ for normalization constraint. This set of probabilities imposes the equality between the expec- 
tations of observable and theirs empirical estimators given by $N$ couples $\left\{\left(X^{i}, c_{k}\right), i=1, \ldots, N \forall k\right\}$. And note that:

$$
\sum_{k=1}^{K+1} \int P\left(X^{i}, c_{k}\right) d X^{i}=1
$$

guarantees the normalization of the probabilities.

The maximum entropy law solution is classically given, taking into account the hypothesis i.i.d., by:

$$
\begin{aligned}
\widehat{P}\left(X^{i}, c_{k}\right) & =\prod_{j=1}^{M} \hat{P}\left(X_{j}^{i}, c_{k}\right) \\
& =\frac{1}{Z^{M}(\lambda)} \cdot \exp \left[-\sum_{j=1}^{M} \sum_{l=1}^{L} \lambda_{l} \phi_{l}\left(X_{j}^{i}, c_{k}\right)\right]
\end{aligned}
$$

where

$$
Z(\lambda)=\sum_{k=1}^{K+1} \sum_{j=1}^{M} \exp \left[-\sum_{l=1}^{L} \lambda_{l} \phi_{l}\left(X_{j}^{i}, c_{k}\right)\right]
$$

is a normalization function and $\lambda=\left[\lambda_{0}, \ldots, \lambda_{L}\right]^{t}$ verifies:

$$
\begin{array}{r}
\frac{\partial \log Z(\lambda)}{\partial \lambda_{l}}=\frac{1}{M} \sum_{k=1}^{K+1} \sum_{\substack{j=1 \\
l=1, \ldots, L}}^{M} \phi_{l}\left(X_{j}^{i}, c_{k}\right) \\
l=1, \ldots
\end{array}
$$

that is to say

$$
\begin{aligned}
& G_{l}(\lambda)= \sum_{k=1}^{K+1} \sum_{j=1}^{M} \phi_{l}\left(X_{j}^{i}, c_{k}\right) \\
& \times \exp \left[-\sum_{l=1}^{L} \lambda_{l} \phi_{l}\left(X_{j}^{i}, c_{k}\right)\right] \\
& l=0, \ldots, L
\end{aligned}
$$

For more developments on the subject, we can refer to $[16,17,18,19]$ and $[20]$. In the following, we will consider the question about the choice of the observable functions $\phi_{l}\left(X^{i}, c_{k}\right)$.

\subsection{Choice of the observable functions}

The observable functions $\left\{\phi_{l}\left(X^{i}, c_{k}\right) l=1, \ldots, L \forall i\right\}$ permit to translate the knowledge of certain characteristics of the variables by means of equations concerning their expectations (a priori information). Knowing that if the number of observable is important, the model will be all the more complex and will require all the more data, we limit to two observables (laws with two parameters $L=2$ ). The observable functions $\phi_{1}\left(X^{i}, c_{k}\right)$ and $\phi_{2}\left(X^{i}, c_{k}\right)$ is chosen such as:

$$
\phi_{1}\left(X^{i}, c_{k}\right)=c_{k} \cdot X^{i}
$$

which shows the correlation between the observation $X^{i}$ and the class $c_{k}$. And:

$$
\phi_{2}\left(X^{i}, c_{k}\right)=X^{i} \log X^{i}
$$

This observable is introduced by El Chakik in [21] like a term of regularization. Then, law with maximum entropy writes:

$\widehat{P}\left(X^{i}, c_{k}\right)=\frac{1}{Z^{M}(\lambda)} \cdot \prod_{j=1}^{M} \exp -\left[\lambda_{1} c_{k} X_{j}^{i}+\lambda_{2} X_{j}^{i} \log X_{j}^{i}\right]$

which is a law of Shannon's entropy form.

To arrive at this stage, we have the tools necessary for modeling information issued from the various sources to be used in the resolution of the multisource fusion problem.

\section{Proposed modeling and fusion algorithms}

\subsection{Maximum entropy algorithm}

To solve the nonlinear equations 19 or 20 , we use a modified Newton-Raphson algorithm. This method consists of expanding $G_{k}$ in Taylor's series around the trivial values $\lambda^{0}$ of the $\lambda^{\prime}$ 's, drop quadratic and higher order terms, and solve resulting linear system iteratively. This algorithm can be summarized as follows:

$$
\begin{aligned}
d_{l} & =G_{l}(\lambda) \text { for } l=0,1,2 \\
& \cong G_{l}\left(\lambda^{0}\right)+\left(\lambda-\lambda^{0}\right)^{t}\left[\operatorname{grad}\left(G_{l}(\lambda)\right)\right]_{\lambda=\lambda^{0}}
\end{aligned}
$$

where

$$
d_{l}=\frac{1}{M} \sum_{k=1}^{K+1} \sum_{j=1}^{M} \phi_{l}\left(X_{j}^{i}, c_{k}\right) \quad l=1, \ldots, L
$$

Noting the vectors $\delta$ and $\nu$ by

$$
\begin{aligned}
\delta & =\lambda-\lambda^{0} \\
\nu & =\left[G_{0}\left(\lambda^{0}\right)-d_{0}, G_{1}\left(\lambda^{0}\right)-d_{1}, G_{2}\left(\lambda^{0}\right)-d_{2}\right]^{t}
\end{aligned}
$$

and the matrix $\mathbf{G}$ by

$$
\mathbf{G}=\left(g_{l m}\right)=\left(\frac{\partial G_{l}(\lambda)}{\partial \lambda_{m}}\right)_{\lambda=\lambda^{0}} l, m=0,1,2
$$

then equation (24) becomes

$$
\mathbf{G} \cdot \delta=\nu
$$

This system is solved for $\delta$ from which we drive $\lambda=\lambda^{0}+\delta$, which becomes the new vector initial vector $\lambda^{0}$ and the iterations continue until $\delta$ becomes appropriately small. See [19] and [20] for more discussions.

\subsection{Entropy adaptive aggregation algo- rithm}

The algorithm proposed calculates the conditional entropies $H\left(c_{k} / X_{k}\right)$ and chooses measurement $M_{k}^{i}$ which, independently of the others, satisfiyes the fusion rule. 
The aggregative approach is the most natural step to solve the multisource data fusion problem, because intuitively we start by seeking the measurement which discriminates more fused measurement that we must obtain. This approach gradually combines all measurements $M_{k}^{i}$ relating to the same decision $c_{k}$ until satisfaction of the combination entropy rule below:

$\forall c_{k} \in Y, X_{k}^{*}=\arg \min _{X^{i} \in X \backslash X^{(p-1)}}\left\{H\left(c_{k} /\left(X^{(p-1)}, X^{i}\right)\right)\right\}$

where $p$ is aggregated measurements number $(p \leqslant N)$ and the symbol $\backslash$ is removal operation. With $X^{(0)}=\emptyset$ and $X^{(p)}=\left(X^{(p-1)}, X^{i}\right)$.

However, in certain cases, a measurement $M_{k}^{i}$ can bring more informations about the decision $c_{k}$ to it than all the others take separately, whereas all these same measurements taken whole satisfied with the combination criterion. To cure this problem, we propose the desaggregative approach [10], [12] which takes into account all measurements $M_{k}^{i}$ of the contingency table relating to the same decision $c_{k}$ for satisfying the combination entropy rule. This approach can summarized as follows:

$\forall c_{k} \in Y, X_{k}^{*}=\arg \min _{X^{i} \in X^{(p+1)}}\left\{H\left(c_{k} /\left(X^{(p+1)} \backslash X^{i}\right)\right)\right\}$

with $X^{(0)}=X$ and $X^{(p)}=\left(X^{(p+1)} \backslash X^{i}\right)$.

The existence of these two possible modes of combination (aggregative and desaggregative) pose a problem of choice, which depends of the quantity $H\left(c_{k} / X\right)$ and the quantities $H\left(c_{k} / X^{i}\right), i=1, \ldots, N$. We might decided to apply one or the other of these two modes of combination according to the value of $H\left(c_{k} / X\right)$, on overall measurements relating to the decision $c_{k}$, compared to a threshold which it would be necessary fixed (difficult). It appears most satisfying to aggregate first bit by bit measurements, and next to test the optimality of fused measurement resulting by applying the composition of the combination modes $A M \circ D M$ and/or $D M \circ A M$ as much as possible until stabilization. Convergence is given by contracting nature of the composition of the combination modes. Finally, we propose the combination rule, that we called entropy adaptive aggregation (EA2) rule, as follows:

$\forall c_{k} \in Y, X_{k}^{E A 2}=\arg \min _{X_{k}^{*}}\left\{H\left(c_{k} / A M \circ D M\left(X_{k}^{*}\right)\right)\right.$
$\left.\odot H\left(c_{k} / D M \circ A M\left(X_{k}^{*}\right)\right)\right\}$

where $X_{k}^{*}$ is initially given by equation 28 and $\odot$ is an operation of type divided and aggregated.
The principle of EA2 algorithm, which is based on the entropy adaptive aggregation rule, is illustrated into Figure 3.

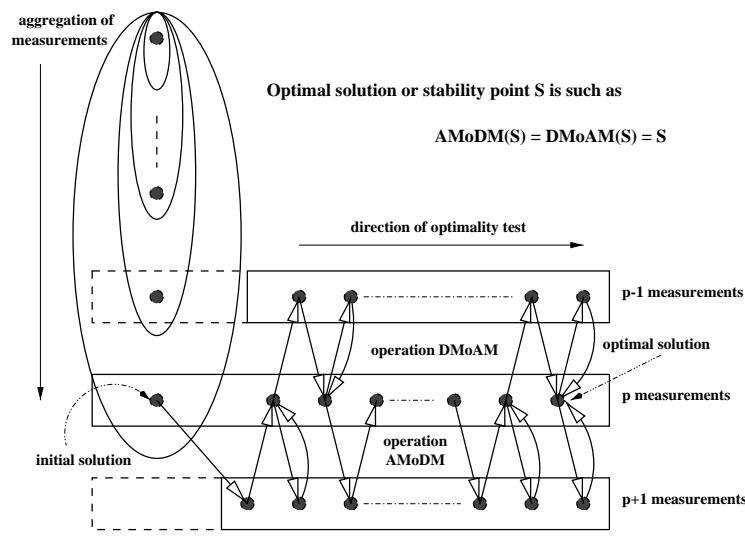

Figure 3: EA2 algorithm principle

In the following section, we describe an application problem where the data fusion made.

\section{$5 \quad$ Entropy model application}

In this section, our aim is to provide briefly a first illustration of entropy model through the application of distributed estimation system with data fusion. More details about this application, the interested reader should refer to future work. We show here describes the use of the two principal aspects of the entropy model describes above (data combination and decision making) in this type of problem.

\subsection{Distributed estimation system with Entropy model}

The application we consider is as follows. Consider the distributed estimation system shown in Figure 1. The system consists of $N$ sensors whose respective measurements, $X^{1}, \ldots, X^{N}$ are related to some unobservable quantity, say $Y$. Each sensor processes its respective measurement and transmits the result to common fusion center. We assume the sensors do not communicate with each other, and there is no feedback from the fusion center to the sensors. The task of the fusion center is to estimate the unobservable quantity $Y$. We denote this estimate by $\widehat{Y}$. Clearly, as indicated in the introduction section, $\widehat{Y}$ is a function of $X^{1}, \ldots, X^{N}$, and can write $\widehat{Y}=f\left(X^{1}, \ldots, X^{N}\right)$ for some function $f$. The problem then is to choose the function $f$ so that $\widehat{Y}$ is close to $Y$ in some sense. We propose the minimum conditional entropy fusion solution as:

$$
\widehat{Y}=\arg \min _{Y_{k}, X_{k}^{E A 2}}\left\{H\left(Y_{k} / X_{k}^{E A 2}\right)\right\}
$$

where $X_{k}^{E A 2}$ is fused information, given by combination entropy rule 30 . 


\subsection{Results and discussion}

We provide here the numerical result of distributed estimation problem to show the effectiveness of the proposed entropy model solution. More complete numerical studies are still under investigation.

\section{Conclusions and future work}

This paper has described the general formalism of entropy method for multisource data fusion, Figure 2 . We have shown as well the importance of the step of information representation for modeling entropy method. We have developed an algorithm that we have called EA2 algorithm for multisource data fusion problem, which is based on the entropy model. We are now investigating alternative domains, such as that of the estimation problem given above, to study the implication of our EA2 algorithm in a data fusion problem. Evaluation of the EA2 algorithm is ongoing, and refinements will continue to be made.

\section{References}

[1] R. C. Luo and M. G. Kay. Data fusion and sensor integration: State-of-the-art 1990s. In M. A. Abidi and R. C. Gonzales, editors, Data Fusion in Robotics and Machine Intelligence, Academic Press, Inc., 1992.

[2] B. Fassinut-Mombot, M. Zribi, and J. B. Choquel. Fusion of information multisensor heterogeneous using an entropy criterion. In Proceedings of the $2^{\text {nd }}$ International Conference on Information $\mathrm{Fu}^{-}$ sion, volume 2, pages 1204-1210, July 1999.

[3] C. E. Shannon. A mathematical theory of communication. Bell System Technical Journal, 27:550553 and 623-656, July and October 1948.

[4] C. E. Shannon and W. Weaver. The Mathematical Theory of Communication. In University of Illinois Press, Urbana, IL, 1949.

[5] A. De Luca and S. Termini. A definition of non-probabilistic entropy in the setting of fuzzy sets theory. Information and Control, 20:301-312, 1972.

[6] I. Couso, P. Gil, and S. Montes. Measure of fuzziness and information theory. In Information Processing and Management of Uncertainty in Knowledge, pages 501-505, July 1996.

[7] A. Fioretto and A. Sgarro. A second step information measure and the uncertainty of bodies of evidence. In Information Processing and Management of Uncertainty in Knowledge, pages 687691, July 1996.

[8] Y. Zhou and H. Leung. Minimum entropy approach for multisensor data fusion. In IEEE Signal Processing Workshop on High-Order Statistics (SPW-HOS'97), pages 336-339, July 1997.
[9] J.R. Quinlan. Learning efficient classification procedures and their application to chess and games, machine learning, an artificial intelligence approach. In 5ème Journées Internationales Analyse des données et Informatique, Tiogra, 1983.

[10] V. M. Toro Cordoba. Contribution à l'Analyse Structurale de systèmes complexes à l'aide de l'entropie et ses généralisations. Thèse de doctorat 3ème cycle, USTLFA, Mars 1982. $N^{\circ} 955$.

[11] Mohammed Barboucha. Modélisation structurale des systèmes complexes, Extraction et validation des règles d'un système expert. Thèse de doctorat d'état, USTLFA, Juin 1987.

[12] Christophe Desrousseaux. Utilisation d'un critère entropique dans les systèmes de détection. Thèse de doctorat 3ème cycle, Université des Sciences et Technologies de Lille, USTL, Décembre 1998.

[13] I. Bloch and H. Maitre. Data fusion in image processing: information models and decisions. Traitement du Signal, 11(6):435-446, 1994.

[14] R. Duda and P. Hart. Pattern classification and scene analysis. Wiley, New-York, January 1973.

[15] R. C. Connant. Detecting Subsystems of a complex System. IEEE Transactions on Systems, Man and Cybernetics, SMC-2(4):550-553, september 1972 .

[16] A. Mohammad-Djafari and J. Idier. Maximum likelihood estimation of the lagrangian parameters of the maximum entropy distributions. In G.J. Erikson C.R. Smith and P.O. Neudorfer, editors, Maximum Entropy and Bayesien Methods, pages 131-140., Printed in the Netherlands, 1991. Kluwer Academic Publishers.

[17] Ali Mohammad-Djafari. Estimation par maximum de vraisemblance des lagrangiens des distributions à maximum d'entropie. In 13ème Colloque sur le Traitement du Signal et des Images, GRETSI, pages 9-12, 1991.

[18] Ali Mohammad-Djafari. Entropy in signal processing. Traitement du Signal, 15(6):545-551, 1998.

[19] A. Mohammad-Djafari. A Matlab program to calculate the maximum entropy distributions. In G.J. Erikson C.R. Smith and P.O. Neudorfer, editors, Maximum Entropy and Bayesien Methods, pages 221-233. Kluwer Academic Publishers, Printed in the Netherlands, 1991.

[20] A. Zellner and R. A. Highfield. Calculation of maximum entropy distributions and approximation of marginal posterior distributions. Journal of Econometrics, 37:195-209, 1988.

[21] Fadi El Chakik. Maximum d'entropie et réseaux de neurones pour la classification. Thèse de doctorat 3ème cycle, Institut Polytechnique de Grenoble, Septembre 1998. 